\title{
Adverse and Benevolent Childhood Experiences Predict Mental Health During the COVID-19 Pandemic
}

\author{
Jenalee R. Doom ${ }^{1}$ (D) $\cdot$ Deborah Seok $^{1} \cdot$ Angela J. Narayan $^{1} \cdot$ Kathryn R. Fox ${ }^{1}$ \\ Accepted: 16 April 2021 / Published online: 23 April 2021 \\ (C) Springer Nature Switzerland AG 2021
}

\begin{abstract}
Adverse childhood experiences (ACEs) are associated with poorer adult mental health, and benevolent childhood experiences (BCEs) are associated with better adult mental health. This study aims to test whether ACEs and BCEs predict adult mental health above and beyond current stress and social support during the COVID-19 pandemic. We analyzed data from undergraduate and graduate students $(N=502)$ at an urban private university in the Western United States. An online survey was conducted to assess ACEs and BCEs, current stress and social support, depressive and anxiety symptoms, perceived stress, and loneliness in May 2020. Higher levels of ACEs were associated with higher levels of depressive symptoms, $\beta=0.45, p=0.002$. Higher levels of BCEs were associated with lower depressive symptoms, $\beta=-0.39, p=0.03$; lower perceived stress, $\beta=-0.26, p=$ 0.002 ; and less loneliness, $\beta=-0.12, p=0.04$. These associations held while controlling for current stress, social support, and socioeconomic status. Childhood experiences are associated with mental health during the COVID-19 pandemic. BCEs should be considered an important promotive factor, independent of ACEs, for psychological well-being during a global public health crisis. BCEs should be included along with ACEs in future research, assessment, and screening with distressed and vulnerable populations.
\end{abstract}

Keywords Adverse childhood experiences · Benevolent childhood experiences · Mental health $\cdot$ COVID-19

\section{Introduction}

The COVID-19 pandemic has led to a mental health crisis in the USA (Czeisler et al., 2020). Social distancing, health and financial concerns, uncertainty, and other stressors have contributed to elevated mental health difficulties, stress, and loneliness. Very little research has been done, however, to understand how a history of childhood adversity or positive experiences may affect mental health during a global health crisis

Jenalee R. Doom

Jena.Doom@du.edu

Deborah Seok

Deborah.Seok@du.edu

Angela J. Narayan

Angela.Narayan@du.edu

Kathryn R. Fox

Kathryn.Fox@du.edu

1 Department of Psychology, University of Denver, 2155 S. Race St, Denver, CO 80210, USA such as the COVID-19 pandemic. The current study aimed to understand whether both childhood adversity and positive childhood experiences predicted mental health in adults during a global pandemic.

Childhood adversities, such as experiencing abuse or neglect or exposure to parental mental illness, are associated with poorer mental health across the lifespan. Specifically, adverse childhood experiences (ACEs) are associated with adult depression, anxiety, suicide, and substance use problems (Choi et al., 2017; Karatekin, 2018; Merrick et al., 2017; Sareen et al., 2013). Examples of ACEs include childhood abuse, neglect, exposure to caregiver mental illness or incarceration, and domestic violence (Felitti et al., 1998). Higher levels of ACEs are also associated with higher levels of stress in adults (Manyema et al., 2018), which itself is part of a mediating pathway linking childhood adversity and adulthood mental health problems (Jones et al., 2018). For college students specifically, higher levels of ACEs are associated with a greater likelihood of deterioration in mental health across time (Karatekin, 2018).

In the context of a global pandemic, higher levels of ACEs may be a risk factor for poorer mental health. Preliminary 
evidence suggests that adults with higher levels of ACEs report higher levels of anxiety and post-traumatic stress disorder (PTSD) symptoms in response to COVID-19 (Guo et al., 2020). Similarly, a recent study suggests that adolescents with higher levels of early life stress are more likely to report higher levels of depressive symptoms during the COVID-19 pandemic, with evidence that higher current stress may explain the association between early life stress and current depressive symptoms (Gotlib et al., 2020).

Childhood adversity is not the only form of early experience that has long-term associations with mental health outcomes (Narayan, Lieberman, \& Masten, 2021). Higher levels of benevolent childhood experiences (BCEs), including growing up with at least one safe caregiver, having one or more close friends, and having a predictable home routine, predict better mental health in adulthood (Crandall et al., 2020; Narayan et al., 2018). Previous work on BCEs has primarily included populations with significant trauma histories (Merrick et al., 2019; Narayan et al., 2018), though recent work has examined BCEs in general populations, including adults with less extensive childhood adversity histories (Crandall et al., 2020, 2019). Research on BCEs conducted in low-income traumatized samples and in community samples both find that ACEs and BCEs typically have independent associations with mental health problems while controlling for one another in the same models (Crandall et al., 2019; Narayan et al., 2018). Studies also find that positive childhood experiences predict better adult health after controlling for concurrent adult resources, such as social support, in addition to adults' ACEs (Bethell et al., 2019).

In addition to their unique associations, it is possible that ACEs and BCEs interact to predict mental health risk. Providing support for this possibility, one study found that BCEs interacted with ACEs, such that higher levels of BCEs buffered against or neutralized the effects of ACEs on negative health outcomes (Crandall et al., 2019). In this same study, however, there was also evidence that for individuals with very high levels of ACEs (four or more), the effects of BCEs on reducing health problems were attenuated, suggesting some limitations of the protective effects of BCEs when ACEs surpass certain thresholds (Crandall et al., 2019). Another study found that positive childhood experiences, assessed more broadly than via Narayan et al.'s (2018) BCEs scale, had a protective effect on depressive symptoms for women who had experienced sexual abuse (Chung et al., 2008).

In the context of the COVID-19 pandemic, it is possible that ACEs could directly predict higher levels of mental health problems, and BCEs could directly predict lower levels of mental health problems, with no interaction (exacerbating or buffering effects) between the two. Another possibility is that COVID-19 is such a pervasive stressor that childhood experiences will not be associated with current mental health problems. Rather, concurrent adult stressors, such as stressors specific to COVID-19, and concurrent social support could be the most salient proximal predictors of current mental health. Indeed, higher levels of current stress are well-established predictors of poorer mental health in adulthood (Campagne, 2019; Hammen, 2005). Greater levels of social support are also associated with better adult mental health (Hefner \& Eisenberg, 2009; Lakey \& Orehek, 2011).

The current study tested whether, during a pervasive current life stressor (the COVID-19 pandemic), higher levels of ACEs and BCEs would each independently predict current mental health when accounted for together and whether higher levels of BCEs would buffer the association between ACEs and mental health problems (i.e., reflecting an interaction between ACEs and BCEs on mental health outcomes). The study also tested whether ACEs and BCEs remained significant predictors of mental health even after controlling for current factors relevant to adult mental health, including higher levels of COVID-related stress, lower levels of concurrent support, and socioeconomic status (SES). Given previous research supporting the salience of each type of childhood and concurrent factor in predicting adult mental health, we hypothesized that greater COVID-19 stress, lower social support, greater ACEs, and lower BCEs would each be associated with higher levels of depressive symptoms, anxiety symptoms, perceived stress, and loneliness. We also hypothesized that there would be an interaction between ACEs and BCEs, such that higher levels of BCEs would attenuate the association between ACEs and depressive symptoms, anxiety symptoms, perceived stress, and loneliness.

\section{Methods}

\section{Overview}

Undergraduate and graduate students from a private university in a western US city were invited to complete a 30-min Qualtrics survey about mental health during the COVID-19 pandemic following a broader institutional research survey assessing student well-being. Participation was completely voluntary, and students who completed the 30-min mental health survey were entered into a gift card raffle, where they had a chance to receive one of several $\$ 50$ gift cards. The survey was administered from May 8 to 18,2020 , while instruction was entirely online.

\section{Participants}

Undergraduate $(N=199)$ and graduate students $(N=303)$ completed questionnaires about ACEs, BCEs, COVID-19 disruptions, current social support, depressive symptoms, anxiety symptoms, perceived stress, and loneliness. Student age 
ranged from 18 to 58 years $(M=25.9, S D=7.62$, median $=$ 24). Most participants reported that they are currently Master's level (47.2\%) and undergraduate level students $(39.4 \%)$. Students identified as $68.1 \%$ female, $21.1 \%$ male, and $10.0 \%$ transgender and gender nonconforming (i.e., gender distinct from the gender assigned at birth). Students identified as the following racial/ethnic backgrounds: $4.8 \%$ Asian, 7.0\% Hispanic/Latino(a/x), 9.2\% Mixed Race, $74.9 \%$ nonHispanic/Latino(a/x) White, and 3.8\% Other. Participants identifying as Black $(n=14)$ and Native American $(n=9)$ were included in the "Other" race/ethnicity category, given the low number of participants identifying in these ways.

\section{Measures}

\section{ACEs}

The presence of 8 individual ACEs was assessed using items from the ACEs scale (Centers for Disease Control and Prevention, 2017; Felitti et al., 1998). Items included in this study were experiences between birth and 18 years of (1) emotional neglect, (2) physical neglect, (3) emotional abuse, (4) physical abuse, (5) parental separation or divorce, and household member (6) substance abuse, (7) mental illness, and (8) incarceration. ACEs have consistently been associated with increased mental and physical health problems in adulthood (Centers for Disease Control and Prevention, 2017; Felitti et al., 1998), indicating good predictive validity. Positively endorsed items were summed for the ACEs score $(M=1.86$, $S D=1.97$, range $=0-8)$, in which higher ACEs scores reflect higher exposure to childhood adversity.

\section{BCEs}

The BCEs scale is a checklist of 10 positive childhood experiences occurring between birth and 18 years (Narayan et al., 2018). Items include (1) having at least one safe caregiver, (2) having at least one good friend, (3) having beliefs that gave comfort, (4) enjoying school, (5) having at least one teacher who cared, (6) having good neighbors, (7) having an adult (not a parent/caregiver) who could provide support or advice, (8) having opportunities to have a good time, (9) having a positive self-concept, and (10) having a predictable home routine. The BCEs scale has demonstrated high test-retest reliability, $r=0.80, p<0.01$, good cultural generalizability, and good predictive validity for later mental health problems (Narayan et al., 2018). Positively endorsed items were summed for a total BCEs score $(M=8.70, S D=1.68$, range $=1-10$ ), in which higher numbers reflect more positive childhood experiences.

\section{COVID-19 Stressors}

COVID-19 stressors were assessed with a checklist including COVID-specific negative experiences adapted from the Epidemic-Pandemic Impacts Inventory (EPII; Grasso et al., 2020). These experiences were drawn from 15 items, including (1) participant contracted COVID-19; (2) family member(s) contracted COVID-19; (3) friend(s) contracted COVID-19; (4) romantic partner(s) contracted COVID-19; (5) participant lost job; (6) parent/guardian lost job; (7) another family member(s) lost job; (8) participant's academic performance deteriorated; (9) participant had difficulty paying bills or buying necessities (e.g., food); (10) participant could not afford rent; (11) trip/big event participant had been looking forward to was canceled; (12) close loved one (not including parent/guardian) died; (13) relative (not including parent/guardian) died; (14) parent/guardian died; and (15) other (asked to write in response), which was coded as an additional stressor. Due to time constraints in the survey, these 15 COVID-specific negative experiences were selected from the longer EPII instrument because they were those most likely to be stressful and disruptive for university students. Positively endorsed stressors were summed for a maximum possible score of $15(M=2.95, S D=1.66$, range $=0-9)$.

\section{Social Support}

Current social support was measured by one broad and multidimensional question from the Adolescent Social Connection \& Coping during COVID-19 Questionnaire (Pfeifer, 2020): "Overall, how well do you feel like the following groups are meeting your social needs in the PAST TWO WEEKS?" Groups included (1) your friends, (2) other peers your age (acquaintances), (3) family members that do not live in your house, (4) romantic interests/partners, and (5) people that do live in your house. Responses ranged from 0 (not at all), 1 (very slightly), 2 (slightly), 3 (moderately), 4 (very well), to 5 (extremely well). The mean of non-missing responses was used. For example, if a student did not have a romantic partner, the mean of the responses for the other groups was used. In this sample, responses ranged from 0 to $5(M=2.81 ; S D=0.97)$. The full questionnaire was not administered due to time restrictions. Items from this questionnaire were chosen because it was specific to COVID-19, and the social support items used for this study were not specific to adolescents.

\section{Depressive Symptoms}

Current depressive symptoms were measured using the 9-item Patient Health Questionnaire (PHQ-9; Kroenke et al., 2001). Participants reported the frequency from 0 (not at all) to 3 (nearly every day) in which they experienced depressive 
symptoms in the past 2 weeks, including depressed mood, lack of pleasure in usual activities, sleep problems, fatigue, low appetite or overeating, and concentration difficulties. The PHQ-9 has demonstrated excellent reliability and validity (Kroenke et al., 2001). Items were summed for a total score of depressive symptoms $(M=10.0, S D=6.55$, range $=0-27, \alpha=$ $0.90)$.

\section{Anxiety Symptoms}

Current anxiety symptoms were measured using the Generalized Anxiety Disorder 7-item scale (GAD-7; Spitzer et al., 2006). Participants reported the frequency from 0 (not at all) to 3 (nearly every day) that they have been bothered by certain problems in the past 2 weeks, such as feeling nervous or on edge, irritable, having trouble relaxing, and feeling afraid that something awful might happen. The GAD-7 has good reliability and validity (Spitzer et al., 2006). Items were summed for a total score of anxiety symptoms $(M=9.4, S D=$ 5.64, range $=0-21, \alpha=0.92$ ).

\section{Perceived Stress}

Current perceived stress was measured with the brief version of the Perceived Stress Scale 4 (PSS-4), which includes four items assessing the participant's inability to manage and cope with stressors in the past month on a scale from (1) never to (5) very often. Items included perceived lack of ability to control the important things in life and that difficulties were piling up and feeling confident in handling personal problems and that things were going well (the latter two were reverse scored). The PSS-4 has adequate reliability and validity (Mitchell et al., 2008; Vallejo et al., 2018). Responses were summed, with higher numbers indicating greater perceived stress $(M=$ $9.38, S D=3.03$, range $=4-16, \alpha=0.81$ ).

\section{Loneliness}

Current loneliness was assessed with the Three-Item Loneliness Scale (Hughes et al., 2004), which draws items from the UCLA Loneliness Scale (Russell et al., 1980) and is rated by frequency from 1 (hardly ever) to 3 (often). Items include (1) lacking companionship, (2) feeling left out, and (3) feeling isolated from others. The Three-Item Loneliness Scale has acceptable reliability and validity, and it is ideal for short surveys (Hughes et al., 2004). Items were summed, with higher scores representing greater feelings of loneliness $(M$ $=5.89, S D=1.91, \alpha=0.78$, range $=3-9$ ).

\section{Covariates}

Covariates included participant age, race/ethnicity, gender, and SES. Age was reported in years. Race/ethnicity was dummy-coded into Mixed Race, Latinx, Asian, and Other, with White as the reference group as it was the option with the largest cell size. Gender was dummy-coded as female, male, or transgender and gender non-conforming (including anyone who indicated a gender distinct from their sex assigned at birth). Subjective SES was self-reported using the MacArthur Scale of Subjective Social Status (Adler \& Stewart, 2007), which asks students to report their status relative to other people in the USA. The scale is a ladder with 10 rungs, with " 10 " being the lowest part of the ladder and " 1 " being the highest. This scale has good reliability and validity for predicting health outcomes (Operario et al., 2004). Participants' subjective social status spanned the full scale (i.e., $1-10)$ with a mean score of $4.76(S D=1.71)$, indicating just above average self-reported SES.

\section{Data Analytic Plan}

Pearson correlations were conducted to examine associations among ACEs, BCEs, COVID-19 stressors, social support, generalized anxiety symptoms, depressive symptoms, perceived stress, loneliness, and subjective SES. Next, multiple linear regressions were conducted to examine whether ACEs and BCEs, COVID-19 stressors, social support, and covariates significantly predicted (a) symptoms of depression, (b) symptoms of generalized anxiety, (c) perceived stress, and (d) loneliness. For each regression, we included the following covariates: subjective SES, age, race, and gender. Finally, we duplicated each regression model including the interaction between ACEs and BCEs, before comparing model fit between the first and second models using analysis of variance.

\section{Results}

\section{Descriptive Statistics}

Pearson correlations across key variables of interest are shown in Fig. 1. Higher ACEs were significantly associated with fewer BCEs, more COVID-19 stressors, greater loneliness, less social support, more perceived stress, greater anxiety and depressive symptoms, and lower subjective SES. Higher BCEs were associated with fewer COVID-19 stressors, less loneliness, greater social support, less perceived stress, fewer anxiety and depressive symptoms, and higher subjective SES. Higher levels of COVID-19 stressors were significantly associated with higher levels of loneliness, lower social support, higher perceived stress, more anxiety and depressive symptoms, and lower subjective SES. Greater social support was associated with lower levels of loneliness, perceived stress, anxiety and depressive symptoms, and higher subjective SES. 
Fig. 1 Pearson correlation matrix for key study variables

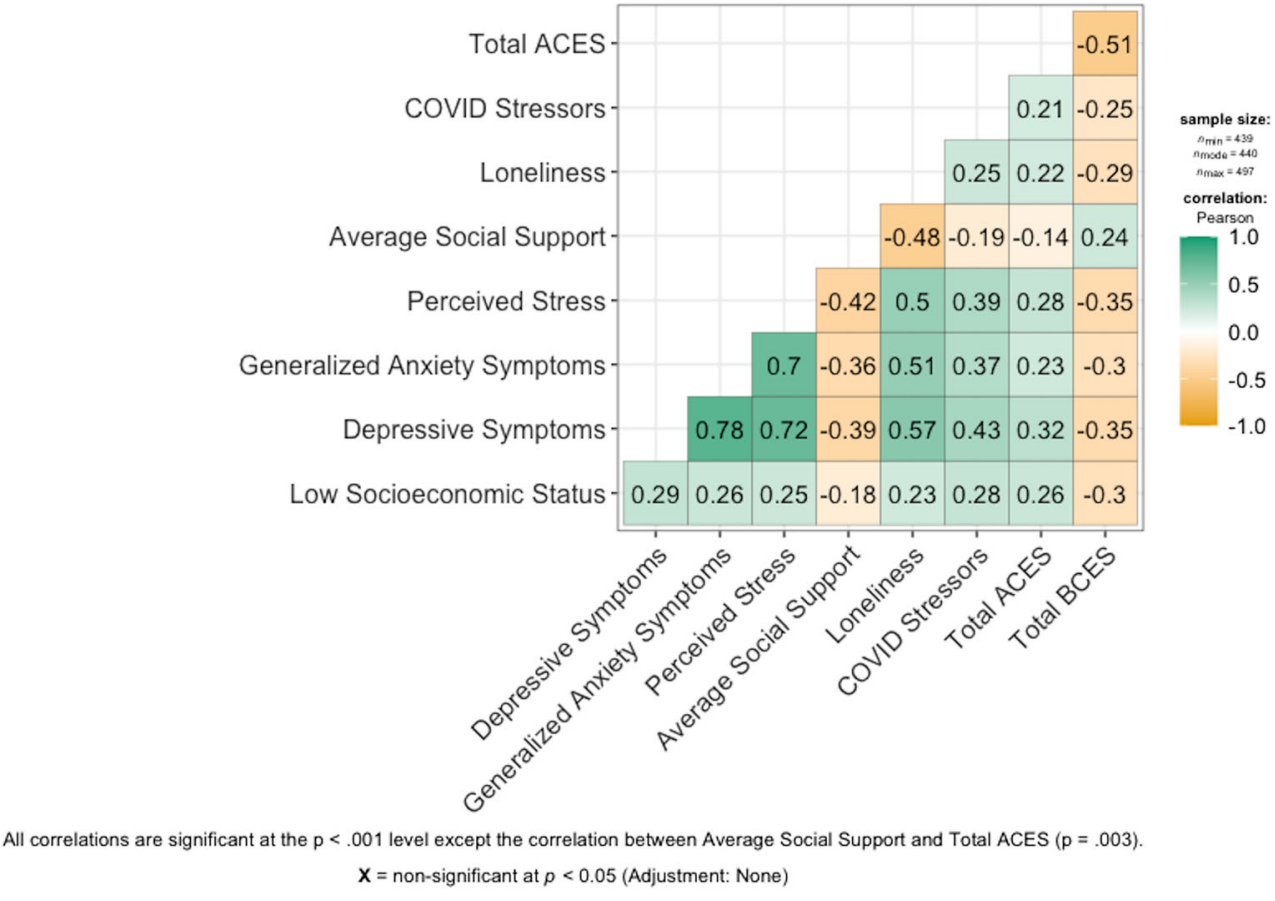

\section{Multiple Linear Regressions}

Detailed results of each linear regression model are depicted in Figs. 2, 3, 4, and 5. In the succeeding text, we describe model fit statistics of each model and individual effects that reached significance $(p<0.05)$.

\section{Depressive Symptoms}

The independent variables from the first model explained a significant proportion of variance in depressive symptom scores, adjusted $R^{2}=0.37, F_{12,418}=20.7, p<0.001$. The second model including the interaction term was not a better fit than the first simpler model ( $p=0.29)$; therefore, we describe results from the first model only. Higher ACEs were associated with greater depressive symptoms, and higher BCEs were associated with lower depressive symptoms (Fig. 2). Higher cumulative COVID-19 stressors were associated with greater depressive symptoms, and higher social support was associated with lower depressive symptoms. In a second model, the interaction of ACEs and BCEs was not significantly associated with depressive symptoms ( $p=$ 0.29 ). Among covariates, only age and SES were significantly associated with depressive symptoms, with younger age and lower SES being associated with higher depressive symptoms.

\section{Generalized Anxiety Symptoms}

The independent variables from the first model explained a significant proportion of variance in generalized anxiety symptom scores, adjusted $R^{2}=0.31, F_{12,416}=16.86, p<0.001$. The second model including the interaction term was not a better fit than the first simpler model ( $p=0.38$ ); therefore, we describe results from the first model only. Neither ACEs nor BCEs were associated with generalized anxiety symptoms (Fig. 3). Higher cumulative COVID-19 stressors were associated with higher generalized anxiety symptoms, and higher social support was associated with lower generalized anxiety symptoms. In a second model, the interaction of ACEs and BCEs was not significantly associated with generalized anxiety symptoms $(p=0.18)$. Among covariates, higher age was associated with lower anxiety symptoms. Participants identifying as Latinx, compared to White, reported higher levels of anxiety symptoms. Participants identifying as male, compared to female, reported significantly lower levels of generalized anxiety symptoms.

\section{Perceived Stress}

The independent variables from the first model explained a significant proportion of variance in perceived stress scores, adjusted $R^{2}=0.35, F_{12,416}=19.95, p<0.001$. The second model including the interaction term was not a better fit than the first simpler model $(p=0.79)$; therefore, we describe results from the first model only. Higher BCEs were associated with lower perceived stress (Fig. 4), while ACEs were not associated with perceived stress. Greater cumulative COVID-19 stressors were associated with higher perceived stress, and higher social support was associated with lower perceived stress. In a second model, the interaction of ACEs and BCEs was not significantly associated with perceived stress $(p=0.79)$. Among covariates, participants identifying as Other race, compared to White, and participants identifying as male, compared to female, reported significantly lower levels of perceived stress. 


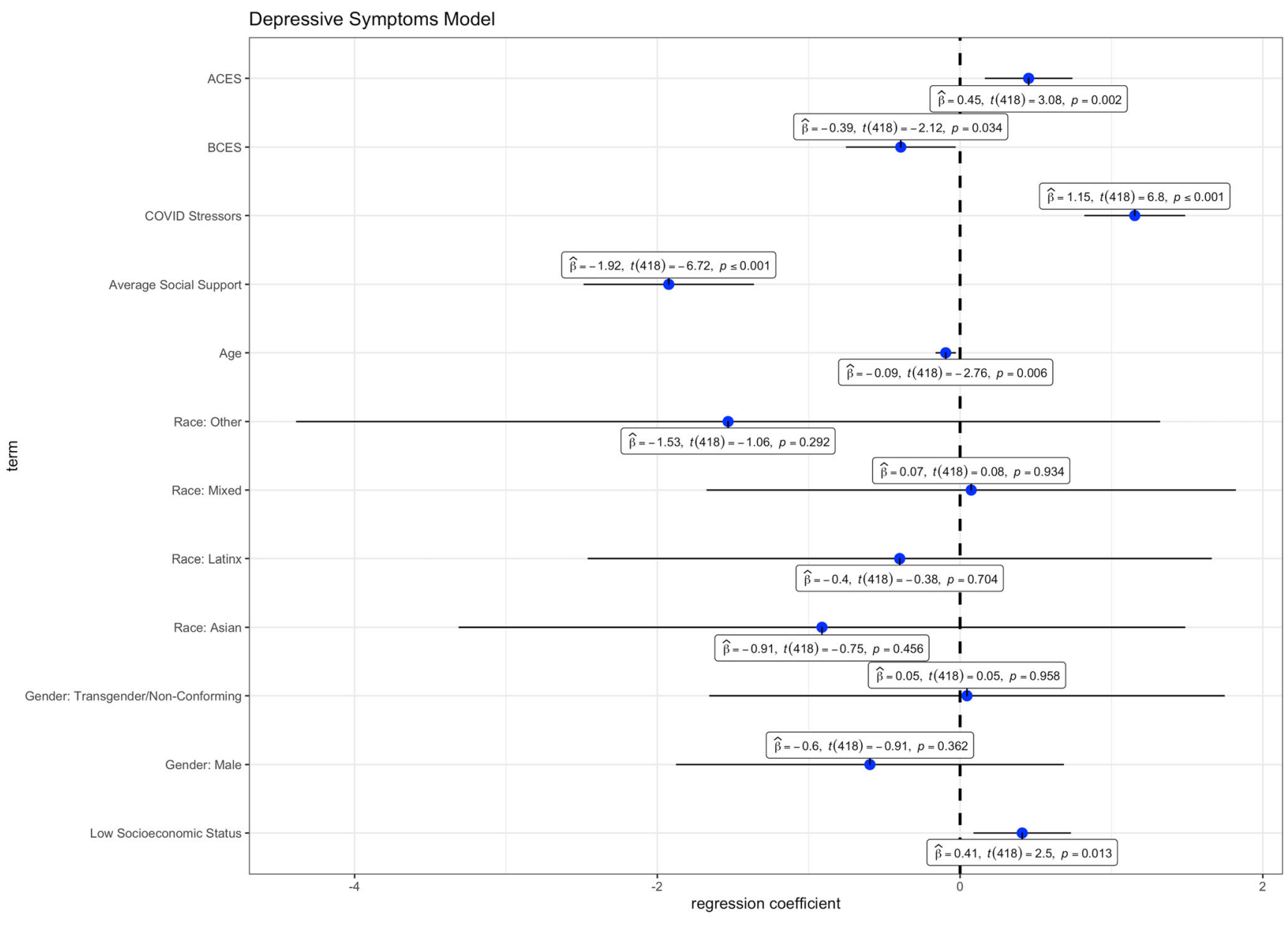

Fig. 2 Multiple linear regression model predicting depressive symptoms

\section{Loneliness}

The independent variables from the first model explained a significant proportion of variance in loneliness scores, adjusted $R^{2}=$ $0.30, F_{12,418}=16.15, p<0.001$. The second model including the interaction term was not a better fit than the first simpler model $(p$ $=0.47$ ); therefore, we describe results from the first model only. Higher BCEs were associated with lower loneliness (Fig. 5), while ACEs were not associated with loneliness. Greater cumulative COVID-19 stressors were associated with higher loneliness, and higher social support was associated with lower loneliness. In a second model, the interaction of ACEs and BCEs was not significantly associated with loneliness $(p=0.47)$.

\section{Discussion}

Current findings contribute to the growing body of research on how the COVID-19 pandemic has affected adults in the USA. Higher levels of ACEs were associated with higher levels of depressive symptoms, and higher levels of BCEs were associated with lower levels of depressive symptoms, perceived stress, and loneliness. These findings support study hypotheses of direct main effects of ACEs and BCEs on mental health problems during COVID-19 and underscore the importance of both positive and negative childhood experiences on long-term mental health outcomes during a global pandemic.

Our findings that childhood experiences were associated with mental health outcomes in adulthood, above and beyond current stressors, are mostly consistent with previous research on the negative effects of ACEs and the positive effect of BCEs. However, the specific mechanisms by which these early experiences affected participants' mental health outcomes are less clear. For example, unlike Gotlib et al. (2020), we did not find a significant association between ACEs and perceived stress after controlling for current COVID-19 stressors. Furthermore, while previous studies have demonstrated the ability of BCEs to neutralize or buffer against the negative effects of ACEs (Crandall et al., 2020, 2019; Narayan et al., 2018), we did not find any evidence of such an interaction in the current study. The lack of interaction could be due to the nature of the sample (low variability and high average BCE scores) or the unique context of the pandemic. The pandemic 


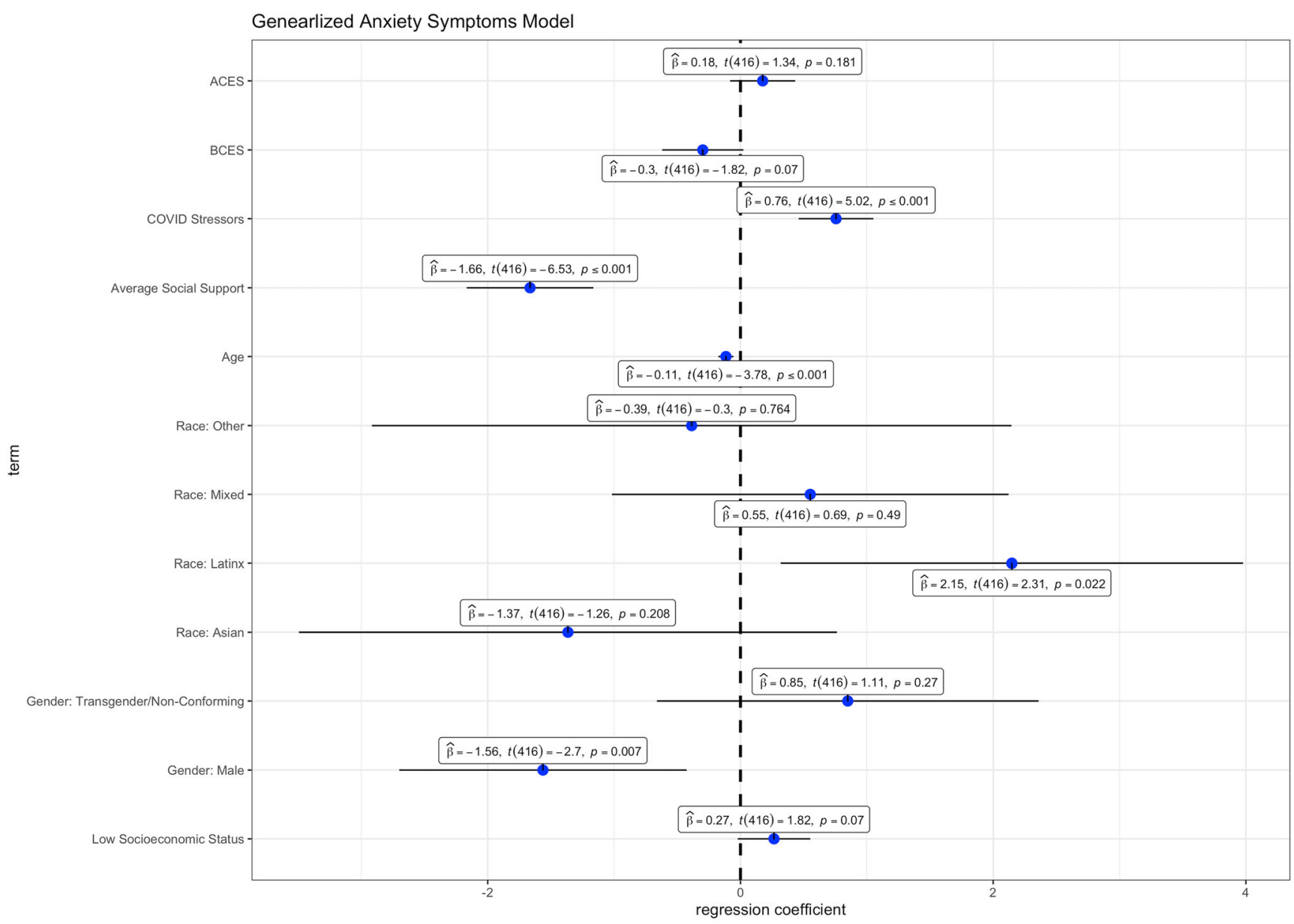

$A I C=2557, B I C=2614$

Fig. 3 Multiple linear regression model predicting generalized anxiety symptoms

is a unique type of stressor that includes fear about the effects of the COVID-19 virus on oneself or others, the emotional toll of social distancing and isolation, and potential economic impacts, among other significant stressors (Pfefferbaum \& North, 2020). It is possible that these additional stressors may contribute to a unique psychosocial context where ACEs and BCEs do not interact but, instead, independently predict mental health. ACEs and BCEs may work independently to confer risk for or directly reduce the risk of mental health problems. Indeed, like other studies in both low-income and community samples (e.g., Crandall et al., 2020; Merrick et al., 2019; Narayan et al., 2018), ACEs and BCEs were only modestly negatively correlated, suggesting that ACEs and BCEs are mostly independent experiences, and the presence of one does not preclude the presence of the others. A further longitudinal investigation is needed to examine whether ACEs and BCEs interact in other samples or other contexts.

Somewhat contrary to hypotheses, BCEs were significantly associated with a broader range of mental health outcomes compared to ACEs. While ACEs were only significantly associated with higher depressive symptoms, BCEs were significantly associated with lower levels of depressive symptoms, perceived stress, and loneliness. This specific finding is encouraging for several reasons. First, while the BCEs scale has been used with high-risk samples such as low-income pregnant women and homeless parents (Merrick et al., 2019; Narayan et al., 2018), more recent studies have demonstrated that BCEs also predict adult health outcomes in lower-risk community samples (Crandall et al., 2020, 2019). The current study further adds to the validation of this measure and confirms the benefits of positive experiences in samples who are not necessarily low income or characterized by high levels of childhood adversity.

Our results highlight that resilience during a global crisis, such as the COVID-19 pandemic, can be leveraged from preexisting positive childhood relationships, experiences, and resources (Masten \& Motti-Stefanidi, 2020). These findings suggest that promotive factors reflected by the BCEs, which themselves do not depend on higher socioeconomic status in the family of origin, may have beneficial effects on later mental health. These results have a number of implications for both adults and children. First, more widespread assessment and screening of BCEs, similar to the increased screening for ACEs in clinical settings, would be beneficial for identifying 


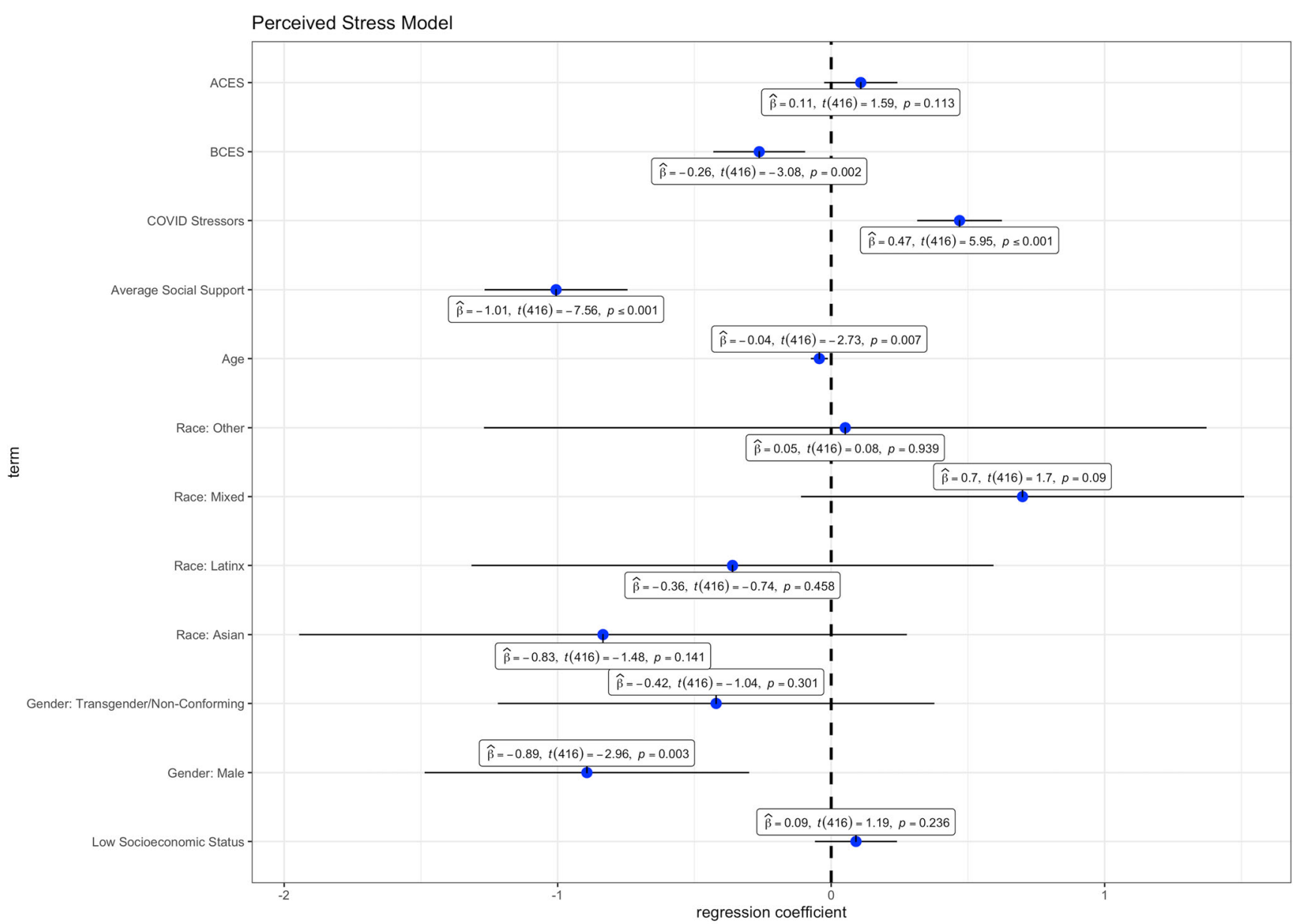

$A I C=1999, B I C=2056$

Fig. 4 Multiple linear regression model predicting perceived stress

those who may be at risk for current or future mental health problems (Narayan et al., 2021). Through screening, adults with low BCEs may be identified as those who may benefit from mental health services to strengthen positive childhood memories with caregivers (Merrick \& Narayan, 2020). Strengthening these memories of childhood positive experiences could also lay the foundation for parents to provide positive relational experiences with their own children in the next generation. Clinical interventions could also be used to strengthen adulthood relationships for those with low BCEs, as current social support was a strong predictor of adult psychosocial outcomes in the current study. Furthermore, these findings also provide support for the idea that building positive relationships and experiences for today's children could enhance their resilience to major stressors in adulthood. This finding is especially important for parents to know as they could be provided with additional resources to increase BCEs in their own children. Parents may also play a role in shaping positive peer relationships or school and neighborhood environments to increase BCEs. These types of interventions may be particularly helpful to deliver during pregnancy or infancy to provide the most benefit as early in development as possible.

The specificity of ACEs in predicting depressive symptoms but not loneliness or perceived stress as BCEs did was somewhat surprising. These findings may be explained by the observation that while ACEs are typically associated with poorer mental health outcomes in adulthood (Karatekin, 2018; Merrick et al., 2017; Sareen et al., 2013), they may be less directly associated with current emotional states, such as perceived stress levels or loneliness. It is possible that higher levels of ACEs are more likely to predict students' mental health problems, whereas higher levels of BCES are more robustly associated with more real-time ability to cope and be less lonely, that is, to draw upon internal or interpersonal resources. This could be due to the fact that BCEs reflect internal and interpersonal resources from childhood (e.g., feeling comfortable with oneself, feeling safe with and close to others). Although warranting replication, mental health problems may be affected by a history of childhood adversity, in addition to an absence of BCEs, whereas higher perceived stress (the perception that stressors are piling up and coping 


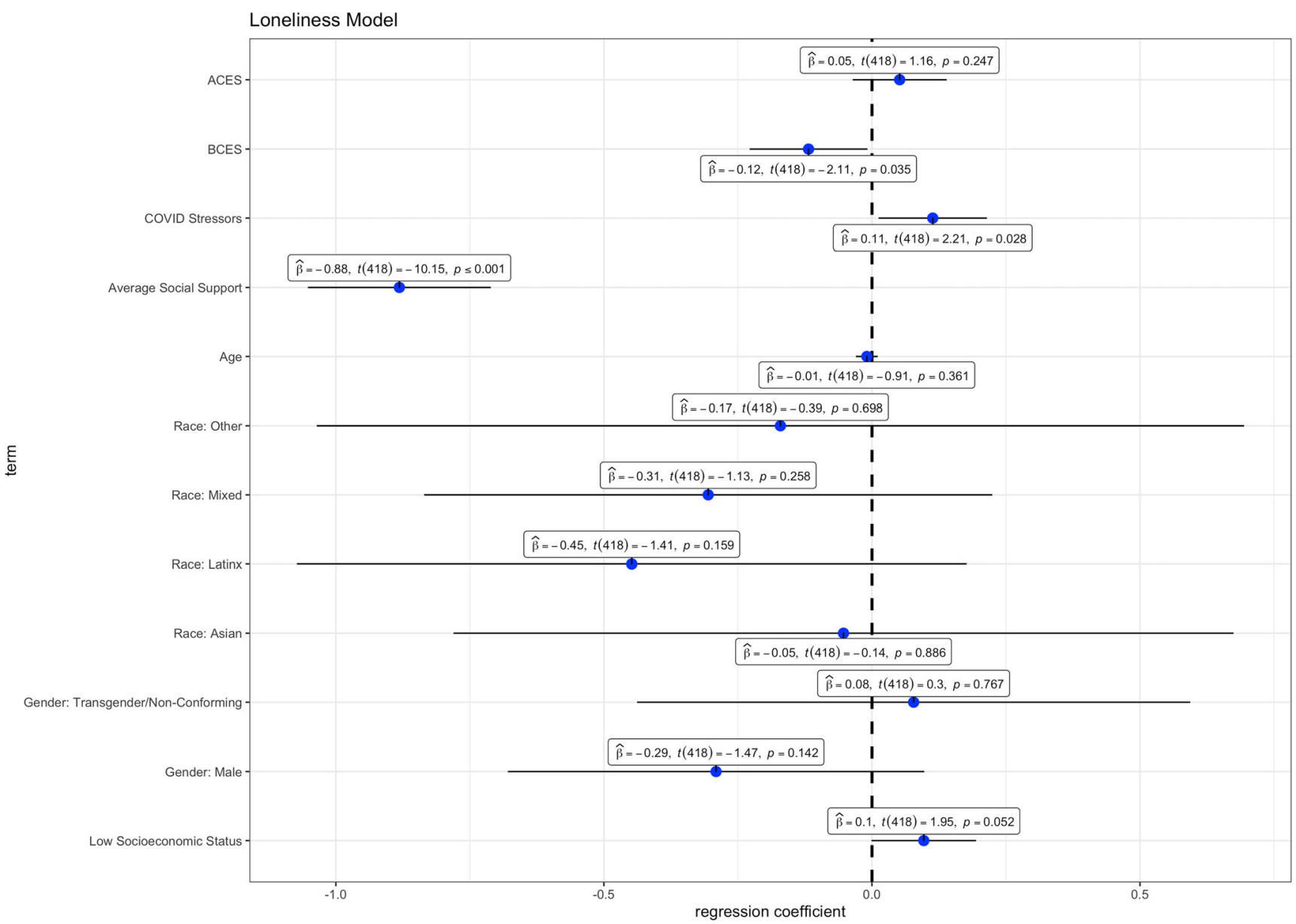

$A I C=1643, B I C=1700$

Fig. 5 Multiple linear regression model predicting loneliness

is difficult) and greater loneliness may be affected by lower levels of childhood resources and positive relationships.

Contrary to hypotheses, neither ACEs nor BCEs predicted anxiety symptoms after controlling for COVID-19 stressors and current social support. There is currently not much evidence linking BCEs or positive childhood experiences to anxiety symptoms, as opposed to depressive and PTSD symptoms (e.g., Bethell et al., 2019; Narayan et al., 2018). Although ACEs have been frequently associated with anxiety symptoms, this association may be smaller than the one between ACEs and depressive symptoms (Racine et al., 2021). In addition, using the GAD-7, anxiety symptoms in our sample $(M=9.4, S D=5.6)$ were higher than in samples of college students before the pandemic ( $M=4.3$, Lee \& Kim, 2019; $M=$ 7.2, Oh et al., 2020). Anxiety was also higher in our sample than in other US university samples during the pandemic $(M=$ 7.8, $S D=6.2$; and $M=7.4, S D=6.0$; Kujawa et al., 2020). Given that our sample reported high anxiety symptoms in the context of the pandemic, COVID-19 stressors and other contextual factors may have been explaining much more of the variance in anxiety symptoms than in samples before the pandemic. To better understand the general link between ACEs, BCEs, and anxiety symptomology, this finding needs to be replicated in other samples both during and after the pandemic.

Both ACEs and BCEs were associated with participants' current social support. In the current study, we found that greater ACEs were associated with lower current social support, consistent with Jones et al.'s (2018) findings, and that higher BCEs were associated with higher current social support. Higher current social support was also associated with lower levels of COVID-19 stressors. We were not surprised to find that current social support was associated with individuals' mental health during the pandemic. Higher current social support was associated with lower levels of depressive and generalized anxiety symptoms, perceived stress, and loneliness, highlighting the importance of having stable and supportive relationships during this challenging time. Results suggest that even amid a major global stressor, social support continues to provide strong mental health and social benefits.

As expected, cumulative COVID-19 stressors were significantly associated with higher levels of depressive and 
generalized anxiety symptoms, perceived stress, and loneliness. We also found that higher ACEs and lower BCEs were associated with higher levels of COVID-19 stressors. Our ACEs finding aligns with previous research that higher levels of ACEs are associated with higher levels of adult adversity (Jones et al., 2018), suggesting that those who experience higher levels of early life stress may be more likely to face increased levels of stressful experiences in adulthood. Our findings may also be explained in part by associations between ACEs and BCEs with other environmental factors that impose greater or lesser risk of experiencing COVID-related challenges and stress. For example, Jones et al. (2018) found that low-income status was significantly associated with both ACEs and adult adversity. Although we did not ask specifically about income status in the current study, we suspect that lower income may underlie several of the ACEs and COVIDrelated challenges we asked about in our surveys, such as students' (or their families') difficulty paying bills and affording rent or student housing. Providing support for this possibility, subjective reports of SES in the present study were associated with ACEs and with adult mental health outcomes, paralleling prior research on this topic (Operario et al., 2004). However, even after controlling for subjective SES, our results linking childhood and current factors remained.

There are several limitations of the current study. First, all study variables were gathered by participant self-report at a single time point, and both ACEs and BCEs were retrospectively reported. Retrospective reports of ACEs have been shown to be valid predictors of adult outcomes in studies that compare retrospective and prospective reports of childhood adversity (Baldwin et al., 2019; Reuben et al., 2016). Further, while more research is needed that compares retrospective versus prospective reports of BCEs, studies have validated the BCEs scale against other assessments of positive childhood experiences, such as positive memory quality (Narayan et al., 2020). Nevertheless, it is possible that memory biases pertaining to participants' personality characteristics or other aspects of their current mental state at the time of survey completion affected their reports across study measures.

Another limitation is that the sample consisted of undergraduate and graduate students from a private university, most of whom identified as White females. The limited scope and diversity of the study's sample restrict the generalizability of our results. Future researchers could certainly benefit from the inclusion of broader populations, including nonstudents and racial or ethnic minorities, in similar studies. Despite low racial/ethnic diversity, the sample included a substantial level of gender diversity, with approximately $10 \%$ identifying with a gender distinct from their gender assigned at birth. This relatively high percentage was surprising and is consistent with some work suggesting increasing acceptance of genderdiverse identities (Bragg et al., 2018). We recommend that future researchers continue to assess gender beyond binary categorization to ensure that these identities can be adequately reflected and represented.

\section{Conclusions}

Study findings provide insight into which individuals are at elevated risk for experiencing poorer mental health during a global crisis and how we can best support them. For instance, findings pertaining to the direct beneficial effects of BCEs and social support on lower levels of mental health problems and loneliness may inform prevention and intervention efforts. These efforts may leverage recollection of positive childhood experiences and existing supportive relationships to promote healthy and adaptive responses to stress, even in samples not intentionally characterized by specific risks (e.g., low income, maltreatment). While there has historically been a strong emphasis on documenting the negative effects of ACEs on longterm health problems, this study highlights the need for more research on how positive childhood experiences may directly promote better health, independent of or despite ACEs. The BCEs items suggest that these positive early experiences need not be extraordinary in nature but that basic resources and favorable relationships within the home, school, and neighborhood settings may have enduring long-term benefits for adult well-being during a global crisis.

Acknowledgements Research reported in this publication was supported by the National Heart, Lung, And Blood Institute of the National Institutes of Health under Award Number K01HL143159 (PI: Doom). The content is solely the responsibility of the authors and does not necessarily represent the official views of the National Institutes of Health. We sincerely thank the students who participated in this study and shared their experiences with us.

Code Availability Code is available by request by contacting the corresponding author.

Data availability Data and materials are available by request by contacting the corresponding author.

\section{Declarations}

Conflict of Interest The authors declare no competing interests.

\section{References}

Adler, N., \& Stewart, J. (2007). The MacArthur Scale of Subjective Social Status. MacArthur Research Network on SES \& Health. Retrieved from Http://Www. Macses. Ucsf. Edu/Research/ Psychosocial/Subjective. Php.

Baldwin, J. R., Reuben, A., Newbury, J. B., \& Danese, A. (2019). Agreement between prospective and retrospective measures of 
childhood maltreatment: A systematic review and meta-analysis. JAMA Psychiatry, 76(6), 584-593.

Bethell, C., Jones, J., Gombojav, N., Linkenbach, J., \& Sege, R. (2019). Positive childhood experiences and adult mental and relational health in a statewide sample: Associations across adverse childhood experiences levels. JAMA Pediatrics, 173(11), e193007-e193007.

Bragg, S., Renold, E., Ringrose, J., \& Jackson, C. (2018). 'More than boy, girl, male, female': Exploring young people's views on gender diversity within and beyond school contexts. Sex Education, 18(4), 420-434.

Campagne, D. M. (2019). Stress and perceived social isolation (loneliness). Archives of Gerontology and Geriatrics, 82, 192-199.

Centers for Disease Control and Prevention. (2017). Adverse childhood experiences (ACEs). Retrieved from https://www.cdc.gov/ violenceprevention/acestudy/

Choi, N. G., DiNitto, D. M., Marti, C. N., \& Segal, S. P. (2017). Adverse childhood experiences and suicide attempts among those with mental and substance use disorders. Child Abuse \& Neglect, 69, 252262.

Chung, E. K., Mathew, L., Elo, I. T., Coyne, J. C., \& Culhane, J. F. (2008). Depressive symptoms in disadvantaged women receiving prenatal care: The influence of adverse and positive childhood experiences. Ambulatory Pediatrics, 8(2), 109-116.

Crandall, A., Miller, J. R., Cheung, A., Novilla, L. K., Glade, R., Novilla, M. L. B., Magnusson, B. M., Leavitt, B. L., Barnes, M. D., \& Hanson, C. L. (2019). ACEs and counter-ACEs: How positive and negative childhood experiences influence adult health. Child Abuse \& Neglect, 96, 104089.

Crandall, A., Broadbent, E., Stanfill, M., Magnusson, B. M., Novilla, M. L. B., Hanson, C. L., \& Barnes, M. D. (2020). The influence of adverse and advantageous childhood experiences during adolescence on young adult health. Child Abuse \& Neglect, 108, 104644.

Czeisler, M. É., Lane, R. I., Petrosky, E., Wiley, J. F., Christensen, A., Njai, R., et al. (2020). Mental health, substance use, and suicidal ideation during the COVID-19 pandemic - United States, June 2430, 2020. Morbidity and Mortality Weekly Report, 69(32), 10491057.

Felitti, V. J., Anda, R. F., Nordenberg, D., Williamson, D. F., Spitz, A. M., Edwards, V., et al. (1998). Relationship of childhood abuse and household dysfunction to many of the leading causes of death in adults: The adverse childhood experiences (ACE) study. American Journal of Preventive Medicine, 14(4), 245-258.

Gotlib, I., Borchers, L., Chahal, R., Gifuni, A., \& Ho, T. C. (2020). Early life stress predicts depressive symptoms in adolescents during the COVID-19 pandemic: The mediating role of perceived stress. Retrieved from https://doi.org/10.31234/osf.io/4dkaf

Grasso, D. J., Briggs-Gowan, M. J., Carter, A. S., Goldstein, B., \& Ford, J. D. (2020). A person-centered approach to profiling COVIDrelated experiences in the United States: Preliminary findings from the Epidemic-Pandemic Impacts Inventory (EPII). Retrieved from https://disasterinfo.nlm.nih.gov/content/files/Epidemic-PandemicImpacts-Inventory-FINAL.pdf

Guo, J., Fu, M., Liu, D., Zhan, B., Wang, X., \& van IJzendoorn, M. H. (2020). Is the psychological impact of exposure to COVID-19 stronger in adolescents with pre-pandemic maltreatment experiences? A survey of rural Chinese adolescents. Child Abuse \& Neglect, 104667.

Hammen, C. (2005). Stress and depression. Annu. Rev. Clin. Psychol., 1, 293-319.

Hefner, J., \& Eisenberg, D. (2009). Social support and mental health among college students. American Journal of Orthopsychiatry, 79(4), 491-499.

Hughes, M. E., Waite, L. J., Hawkley, L. C., \& Cacioppo, J. T. (2004). A short scale for measuring loneliness in large surveys: Results from two population-based studies. Research on Aging, 26(6), 655-672.
Jones, T. M., Nurius, P., Song, C., \& Fleming, C. M. (2018). Modeling life course pathways from adverse childhood experiences to adult mental health. Child Abuse \& Neglect, 80, 32-40.

Karatekin, C. (2018). Adverse childhood experiences (ACEs), stress and mental health in college students. Stress and Health, 34(1), 36-45.

Kroenke, K., Spitzer, R. L., \& Williams, J. B. (2001). The PHQ-9: Validity of a brief depression severity measure. Journal of General Internal Medicine, 16(9), 606-613.

Kujawa, A., Green, H., Compas, B. E., Dickey, L., \& Pegg, S. (2020). Exposure to COVID-19 pandemic stress: Associations with depression and anxiety in emerging adults in the United States. Depression and anxiety, 37(12), 1280-1288.

Lakey, B., \& Orehek, E. (2011). Relational regulation theory: A new approach to explain the link between perceived social support and mental health. Psychological Review, 118(3), 482-495.

Lee, B., \& Kim, Y. E. (2019). The psychometric properties of the Generalized Anxiety Disorder scale (GAD-7) among Korean university students. Psychiatry and Clinical Psychopharmacology, 29(4), 864-871.

Manyema, M., Norris, S. A., \& Richter, L. M. (2018). Stress begets stress: The association of adverse childhood experiences with psychological distress in the presence of adult life stress. BMC Public Health, $18(1), 835$.

Masten, A. S., \& Motti-Stefanidi, F. (2020). Multisystem resilience for children and youth in disaster: Reflections in the context of COVID19. Adversity and Resilience Science, 1, 95-106.

Merrick, J. S., \& Narayan, A. J. (2020). Assessment and screening of positive childhood experiences along with childhood adversity in research, practice, and policy. Journal of Children and Poverty, 26, 269-281.

Merrick, M. T., Ports, K. A., Ford, D. C., Afifi, T. O., Gershoff, E. T., \& Grogan-Kaylor, A. (2017). Unpacking the impact of adverse childhood experiences on adult mental health. Child Abuse \& Neglect, $69,10-19$.

Merrick, J. S., Narayan, A. J., DePasquale, C. E., \& Masten, A. S. (2019), Benevolent childhood experiences (BCEs) in homeless parents: A validation and replication study. Journal of Family Psychology, 33(4), 493-498.

Mitchell, A. M., Crane, P. A., \& Kim, Y. (2008). Perceived stress in survivors of suicide: Psychometric properties of the Perceived Stress Scale. Research in Nursing \& Health, 31(6), 576-585.

Narayan, A. J., Rivera, L. M., Bernstein, R. E., Harris, W. W., \& Lieberman, A. F. (2018). Positive childhood experiences predict less psychopathology and stress in pregnant women with childhood adversity: A pilot study of the benevolent childhood experiences (BCEs) scale. Child Abuse \& Neglect, 78, 19-30.

Narayan, A. J., Atzl, V. M., Merrick, J. S., Harris, W. W., \& Lieberman, A. F. (2020). Developmental origins of ghosts and angels in the nursery: Adverse and benevolent childhood experiences. Adversity and Resilience Science, 1, 121-134.

Narayan, A. J., Lieberman, A. F., \& Masten, A. S. (2021). Intergenerational transmission and prevention of adverse childhood experiences (ACEs). Clinical Psychology Review, 85, 101997

Oh, H. Y., Marinovich, C., Jay, S., Zhou, S., \& Kim, J. H. (2020). Abuse and suicide risk among college students in the United States: Findings from the 2019 Healthy Minds Study. Journal of Affective Disorders, 282, 554-560.

Operario, D., Adler, N. E., \& Williams, D. R. (2004). Subjective social status: Reliability and predictive utility for global health. Psychology \& Health, 19(2), 237-246.

Pfefferbaum, B., \& North, C. S. (2020). Mental health and the COVID-19 pandemic. New England Journal of Medicine, 383(6), 510-512.

Pfeifer, J. H. (2020). Adolescent Social Connection \& Coping during COVID-19 Questionnaire (ASC). Retrieved from https://osf.io/ jakg5/ 
Racine, N., Devereaux, C., Cooke, J. E., Eirich, R., Zhu, J., \& Madigan, S. (2021). Adverse childhood experiences and maternal anxiety and depression: A meta-analysis. BMC psychiatry, 21(1), 1-10.

Reuben, A., Moffitt, T. E., Caspi, A., Belsky, D. W., Harrington, H., Schroeder, F., Hogan, S., Ramrakha, S., Poulton, R., \& Danese, A. (2016). Lest we forget: Comparing retrospective and prospective assessments of adverse childhood experiences in the prediction of adult health. Journal of Child Psychology and Psychiatry, 57(10), 1103-1112.

Russell, D., Peplau, L. A., \& Cutrona, C. E. (1980). The revised UCLA Loneliness Scale: Concurrent and discriminant validity evidence. Journal of Personality and Social Psychology, 39(3), 472-480.
Sareen, J., Henriksen, C. A., Bolton, S.-L., Afifi, T. O., Stein, M. B., \& Asmundson, G. J. G. (2013). Adverse childhood experiences in relation to mood and anxiety disorders in a population-based sample of active military personnel. Psychological Medicine, 43(1), 73-84.

Spitzer, R. L., Kroenke, K., Williams, J. B., \& Löwe, B. (2006). A brief measure for assessing generalized anxiety disorder: The GAD-7. Archives of Internal Medicine, 166(10), 1092-1097.

Vallejo, M. A., Vallejo-Slocker, L., Fernández-Abascal, E. G., \& Mañanes, G. (2018). Determining factors for stress perception assessed with the Perceived Stress Scale (PSS-4) in Spanish and other European samples. Frontiers in Psychology, 9, 37. 